according to functional analyzes reduce the function of the ABCG2 protein (2). Based on in silico prediction, the impact on reduced function is expected for variants p.M71V, p.G74D, p.M131I, p.R147W, p.I242T, p.F373C, p.T434M, p.S476P and p.S572R.

Conclusion: Our data suggest that the common variant p.Q141K and most of the rare variants in the $A B C G 2$ gene affect the function of the ABCG2 urate transporter and are a genetic risk factor for hyperuricemia and gout. REFERENCES:

[1] Stiburkova B, et al. Functional non-synonymous variants of ABCG2 and gout risk. Rheumatology (Oxford). 2017 Nov 1; 56(11):1982-1992.

[2] Toyoda $Y$, et al. Functional characterization of clinically-relevant rare variants in ABCG2 identified in a gout and hyperuricemia cohort. Cells. 2019 Apr $18 ; 8(4)$.

Acknowledgements: This study was supported by the project for conceptual development of research organization 00023728 (Institute of Rheumatology) and RVO VFN64165.

Disclosure of Interests: None declared

DOI: 10.1136/annrheumdis-2021-eular.1799

\section{POS0353 DEEP PHENOTYPING OF PSORIASIS BASED ON LIPID METABOLISM RELATED GENES BY INTEGRATIVE SYSTEMS ANALYSIS}

J. Shi ${ }^{1,2,3}$, S. X. Zhang ${ }^{1,2,3}$, J. Qiao ${ }^{1,2,3}$, S. Song ${ }^{1,2,3}$, R. Zhao ${ }^{1,2,3}$, P. Zhao ${ }^{4}$, G. Y. Liu ${ }^{1,2,3}$, J. Luo ${ }^{1,2,3}$, P. F. He ${ }^{5}$, X. Li ${ }^{1,2,3} .{ }^{1}$ The Second Hospital of Shanxi Medical University, Department of Rheumatology, Taiyuan, China; ${ }^{2}$ Shanxi Li Xiaofeng Medical Groups, Department of Rheumatology, Taiyuan, China; ${ }^{3}$ Key Laboratory of Cellular Physiology at Shanxi Medical University, Ministry of Education, Taiyuan, China: ${ }^{4}$ Shanxi Provincial People's Hospital, Department of Dermatology, Taiyuan, China; ${ }^{5}$ Shanxi Medical University, Medical Data Sciences, Taiyuan, China

Background: Psoriasis is an immune-mediated inflammatory, genetic disease with numerous concomitant metabolic disorders ${ }^{1}$. Patients with psoriasis experience substantial morbidity and increased rates of cardiometabolic diseases ${ }^{2}$. Among the metabolic disorders, lipid disturbances are recognized as a very important part in the pathogenesis of psoriasis and psoriasis-associated cardiometabolic diseases ${ }^{34}$

Objectives: To characterize the molecular features of psoriasis by the development of a classification system that was based on the gene expression profile of lipid metabolism related genes.

Methods: We performed molecular subtyping and association analysis of psoriasis from Gene Expression Omnibus (GEO). The training sets includes GSE13355 cohort $(\mathrm{N}=58)$, GSE14905 $(\mathrm{N}=28)$ cohort and GSE30999 cohort $(N=81)$, while the GSE54456 $(N=92)$ was selected as the validation set. Energy metabolism-related genes were collected from MSigDB (version 7.0; $\mathrm{http}: / /$ software.broadinstitute.org/gsea/msigdb/). Based on these psoriasis lipid metabolism-related genes, we conducted consensus molecular subtyping with nonnegative matrix factorization (NMF). Finally, Gene set variation analysis (GSVA) was used to score individual subtypes against the metabolism-related gene sets, and each group got a GSVA index.

Results: Total 104 lipid metabolism-related genes were clustered into three subgroups (Figure 1A). GSVA revealed the lipid metabolism with the correlation in subtypes. There was no statistical difference was identified between the subtype 1 and subtype $2(P>0.05$, Figure $1 B)$, so we merged the two types as a new Sub1, another subtype was named as the new Sub2 (Figure 1C). We finally identified 2 distinct subtypes of psoriasis in GEO cohort that were characterized by significantly different lipid metabolic gene score. Sub1 was featured by higher metabolic gene score compared with that of Sub2 $(P<0.001 ;$ Figure 1D). The lipid metabolic subtype classification was successfully validated in another independent patient cohorts (GSE54456).

Conclusion: This study established a new classification based on the gene expression profiles of lipid metabolic genes, thereby furthering the understanding of the pathogenesis and psoriasis-associated diseases.

REFERENCES:

[1] Boehncke WH, Schon MP. Psoriasis. Lancet 2015;386(9997):983-94. doi: 10.1016/S0140-6736(14)61909-7 [published Online First: 2015/05/31]

[2] Armstrong AW, Read C. Pathophysiology, Clinical Presentation, and Treatment of Psoriasis: A Review. JAMA 2020;323(19):1945-60. doi: 10.1001/ jama.2020.4006 [published Online First: 2020/05/20].

[3] Goolam Mahyoodeen N, Crowther NJ, Pillay L, et al. Relationship of Visceral Fat and Adipokines with Cardiometabolic Diseases in Psoriasis. Acta Derm Venereol 2019;99(13):1218-23. doi: 10.2340/00015555-3327 [published Online First: 2019/10/04]

[4] Pietrzak A, Michalak-Stoma A, Chodorowska G, et al. Lipid disturbances in psoriasis: an update. Mediators Inflamm 2010;2010 doi: 10.1155/2010/535612 [published Online First: 2010/08/14].
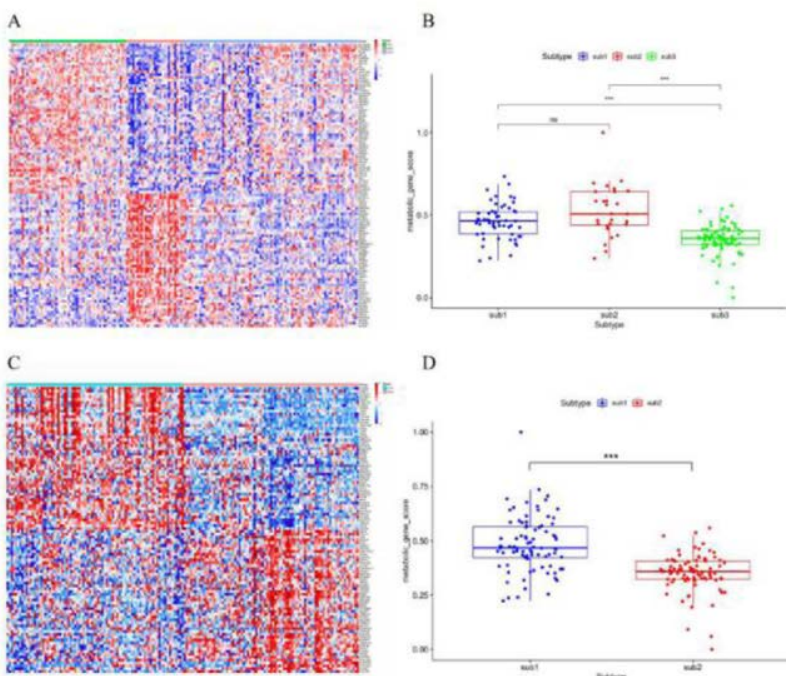

D

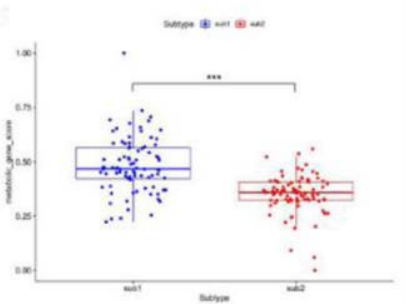

Figure 1: Molecular typing of patients with psoriasis based on lipid metabolism-related gene expression profiles. (A, C) consensus clustering of 167 GEO samples by NMF, a heatmap displaying the expression pattem of the lipid metabolism related subtypes is shown. (B) comparison of lipid metabolic score levels among three subgroups. (D) comparison of lipid metabolic score levels between the final two subgroups. $\mathrm{m} P>0.05 ; * * * \mathrm{P}<0.001$.

Acknowledgements: This project was supported by National Science Foundation of China (82001740), Open Fund from the Key Laboratory of Cellular Physiology (Shanxi Medical University) (KLCP2019) and Innovation Plan for Postgraduate Education in Shanxi Province (2020BY078).

Disclosure of Interests: None declared

DOI: 10.1136/annrheumdis-2021-eular.2073

\section{POS0354 DETECTION OF ABCG2 VARIANTS IN ENCODING OF URATE TRANSPORTERS ASSOCIATED WITH THE} HYPERURICEMIA IN HAEMODIALYSIS PATIENTS

H. Ciferska ${ }^{1,2}$, K. Pavelcova ${ }^{1,2}$, J. Vachek ${ }^{3,4}$, B. Stiburkova ${ }^{5,6} .{ }^{1}$ Institute of Rheumatology, Institute of Rheumatology, Prague, Czech Republic; ${ }^{2}$ First Faculty of Medicine, Charles University, Department of Rheumatology, Prague, Czech Republic; ${ }^{3}$ General University Hospital, Prague and the First Medical Faculty, Charles University, Department of Nephrology, Prague, Czech Republic; ${ }^{4}$ Klatovska Hospital, Dialysis Ward, Klatovy, Czech Republic; ${ }^{1}$ Institute of Rheumatology, Institute of Rheumatology, Prague, Czech Republic; ${ }^{6}$ First Faculty of Medicine, Charles University and General University Hospital in Prague, Department of Pediatrics and Adolescent Medicine, Prague, Czech Republic

Background: Hyperuricemia is associated with gout, hypertension, cardiovascular diseases and renal disease. The presence of chronic kidney disease (CKD) is associated with low excretion of the uric acid as the homeostasis in maintaining of serum levels of uric acid is impaired. Progression of CKD is connected to hyperuricemia and lowering levels of the uric acid is one of the most importan goals in clinical treatment. Dysfunctional variants of ATP-binding cassette transporter subfamily G member 2 (ABCG2), a major urate transporter in the kidney and intestine, are the major causes of hyperuricemia and gout.

A recent studies found that $A B C G 2$ is also a major transporter of uremic toxins and ABCG2 dysfunctional variants are risk genetics factors for mortality in hemodialysis patients.

Objectives: The aim of the present study was to investigate the frequencies of ABCG2 variants in cohort of hemodialysis patients.

Methods: In this retrospective one centre study 167 patients (age $=79,8 \pm 11,03$; female $=74$ ) with end-stage CKD (pre-dialysis $n=86$; dialysis $n=79$ ) were collected Peak urate levels were $456,3 \pm 113,6 \mu \mathrm{mol} / \mathrm{l}$ in pre-dialysis and $572,93 \pm 114,56$ $\mu \mathrm{mol} / \mathrm{l}$ in dialysis. ABCG2 coding regions were analyzed from genomic DNA, as we described previously (1). The reference sequence was defined as version ENST00000237612.7, the reference protein sequence was defined as Q9UNQ0. The chi-square goodness-of-fit test was used to compare minor allele frequencies (MAF), and the log-rank test was used to compare empirical distribution functions.

Results: In the CKD cohort, 15 intronic and seven non-synonymous allelic exonic variants were detected: two common (rs2231137/p.V12M; rs2231142/p Q141K), five ultra rare and/or rare (rs142634180/p.R45Q, rs759726272/p.M131I, rs 140207606/p.R236X, rs138606116/p.G354R, rs138892154/p.A607V), and one novel (p.E344D). Common variant p.V12M, previously reported as protective 